Original Research Paper

\title{
Iris Recognition Using Gauss Laplace Filter
}

\author{
Romany F. Mansour \\ Department of Mathematics, Faculty of Science, (at New Valley), Assiut University, Egypt
}

\author{
Article history \\ Received: 25-03-2016 \\ Revised: $10-06-2016$ \\ Accepted: 15-09-2016 \\ Email: romanyf@aun.edu.eg
}

\begin{abstract}
Biometrics deals with recognition of individuals based on their behavioral or biological features. The recognition of IRIS is one of the newer techniques of biometrics used for personal identification. It is one of the most widely used and reliable technique of biometrics. In this study a novel approach is presented for IRIS recognition. The proposed approach uses Gauss Laplace filter to recognize IRIS. The proposed approach decreases noise to the maximum extent possible, retrieves essential characteristics from image and matches those characteristics with data in a database. This method will be effective and simple and can be implemented in real time. The experiments are carried out using the images of IRIS acquired from a database and MATLAB application has been applied for its effective and simple manipulation of IRIS image. It was observed that developed approach has more accuracy and a relatively quicker time of execution than that of the existing approaches.
\end{abstract}

Keywords: IRIS Recognition, Gauss Laplace Filter, Biometrics

\section{Introduction}

The IRIS is internal organ of the body that is readily visible from outside. Its main aim is to manage the amount of light that enters eye through pupil using dilator and sphincter muscles to manage the size of pupil. It is comprised of elastic fibrous tissue that provides it a very complex pattern of texture. The pattern of texture has no connection with individual's genetic structure and is produced by chaotic processes. The IRIS recognition technique is an important solution of biometrics for people identification. Unlike fingerprint identification, IRIS is more secure (Alotaibi and Hebaishy, 2014). In the recent years several researchers have come up with several techniques for IRIS detection. This research will make use of Gauss Laplace algorithm for accomplishing the same. The study of Chouhan and Shukla (2010) presented a novel statistical feature approach by using Gaussian filter laplacian to recognition of iris. Their aim is to evolve good algorithm that develops the images of iris and matches those characteristics with data in the database of iris. In the study of Savithiri and Murugan (2010) Gaussian pyramid compression technique is used to compress the image of eye and this compressed eye is used for outer and inner boundaries localization of iris region. Located iris is retrieved from the compressed image of eye and after enhancement and normalization it is indicated by a set of data. With Gaussian pyramid compression improved performance of matching is examined down to $0.25 \mathrm{bpp}$ attributed to reduction of noise without an essential texture loss. Schmid et al. (2006) has stated that the algorithm proposed in finds the biometric system optimization on a larger data set on Gaussian model basis acquired from a little data set. Ma et al. (2002) have mentioned that in the Gauss Laplace algorithm the pupil locations and the lower and upper eyelids are predicted using edge detection. This is carried out after the actual image of IRIS has been down sampled by a two factor in every direction which makes it one of the most superior techniques for IRIS detection. Tisse et al. (2003) has mentioned that there are four steps involved in recognition of IRIS. The first step comprises of preprocessing. Then the type and size of the picture is estimated in order to be consequently process them. After that the IRIS texture is retrieved. Lastly the coded image is compared with already coded IRIS to predict the most accurate match. In this study the researcher will apply a combination of the best approaches in every stage of the IRIS detection to obtain the most accurate outcome possible.

\section{Literature Review}

The study of Miyazawa et al. (2008) presented an effective algorithm for IRIS recognition using the 
technique of phase based image matching of image matching using components of phase in two dimensional Discrete Fourier Transform (DFT) of given images. The evaluation of experiments using the database of CASIA IRIS image and ICE 2005 (IRIS challenge evaluation) database clearly explained that the phase components use of IRIS images made it feasible to accomplish highly accurate IRIS recognition with a simple matching algorithm. In order to decrease the data size and to increase the IRIS image visibility the author introduced the notion of two dimensional Fourier Phase Code (FPC) for indicating the information of IRIS.

Durai and Karnan (2010) proposed a study on recognition of IRIS using changed hierarchical phase based matching technology. The suggested system was configured for recognizing IRIS using database of training as input which consists an IRIS for each individual. The final decision was made by HPM as the matching score level architecture in which vectors of featurewere made independently for query images and then compareto enrollment templateswhere each subsystem evaluated its own score of matchingIn the proposed technique the phase components use in 2D IRIS images of Discrete Fourier Transform (DFT) made feasible to achieve highly resilientIRIS recognitionin a distinct way with a simple algorithm of matching.

According to the study of Verma et al. (2012) recognition of IRIS is an accurate and reliable system for biometric identification. In this study the author used Daughman's algorithm segmentation process for recognition of IRIS. The images of IRIS are chosen from the database of CASIA then the pupil and IRIS boundary are predicted from the remaining eye image, removing noises. The segmented region of IRIS was normalized to reduce the inconsistencies of dimension between IRIS areas by using Daugman's Rubber sheet model. Then the characteristics of IRIS were encoded by convolving the normalized IRIS region with a single dimensional filter of $\log$ Gabor and phase quantizing the outcomes to produce bit wise template of biometrics.

In the study of Chitte et al. (2012) an IRIS image synthesis process based on principal component analysis, independent component analysis and Daugman's rubber sheet structure was proposed. The aim of their study was to implement a prototype of working of the techniques and methods used for recognition of IRIS. Their algorithm was proven to be highly reliable and accurate over 200 billion comparisons. In the study the authors compared the outcomes of all 3 algorithms and recognized the best technology used for IRIS recognition.

The study of Jayachandra and Reddy (2013) focused mainly on pupil to identify the eye. To find the edges of image this study proposed the process of canny edge detection to reduce the noisy data in image and find the edges. After finding the edges those images were stored in CASIA database. Secondly the k-means algorithm was used in this study to recognize the edge image of nearest pupil from database for the given input image. The outcomes of this study revealed that recognizing pupil was a better process to identify the eye and raising the accuracy of recognition.

Yao et al. (2014) proposed a study on IRIS feature extraction based on Haar Wavelet transform. In order to, increase the IRIS recognition system accuracy, this study suggested an effective algorithm for feature extraction of IRIS based on two dimensional Haar wavelet transformations. Firstly the image of IRIS was decomposed by two dimensional Haar wavelet 3 times and then a 375 bit code of IRIS was acquired by quantizing entire high frequency co-efficient at 3rd lever. Lastly the authors used a function of similarity degree as the scheme of matching. The outcomes on IRIS database of CASIA revealed that their algorithm has motivating Correct Recognition Rate (CRR) which was nearly 93.18\% accompanying with reduced Equal Error Rate (EER) with $0.54 \%$.

\section{Design and Implementation}

The design and implementation section provides the design of IRIS detection system using Gaussian Laplace filters. Database used for this particular research is CASIA-Iris-Thousand. Information for the database can be seen in this website (http://biometrics.idealtest.org/dbDetailForUser.do?id=4).

The goal of this IRIS recognition system is to acquire HD images of IRIS either from pre-collected images or IRIS scanner. These images clearly reveal the complete eye particularly the pupil and IRIS section. In this particular research the IRIS HD images are acquired using pre collected images that are stored in a database. The Fig. 1 shows the flowchart of the implementation steps.

\section{Segmentation}

Iris is an eye part that encloses the pupil. There are many ways to isolate the IRIS from the eye image. This study uses Hough transform for localization and model of Dugman rubber sheet for normalization then this study uses two varied methods of feature extraction one is log Gabor wavelet for analysis of phase and second is the Gaussian filter Laplacian for statistical analysis. The Hough transform is considered as a strong component in edge linking for line extraction. Its major advantages are its ability to extract lines and insensitivity to noise in areas with pixel gaps. The Hough transform can be used to predict circles, lines or other parametric curves. The purpose is to predict the lines location in images. The benefits of Hough transform are simple implementation, simple conceptually, can be adapted to several forms and 
manage occluded and missing information easily. For instance in hough transform a circle in the ab-plane is presented by (Joshi, 2004):

$$
(a-x)^{2}+(b-y)^{2}=z^{2}
$$

Data was filtered through gabor convolution. Then data is implemented through linear hough transform. Iris is detected through canny edge detection. Then image is compared with the help of phase demodulator and finally calculation is made through calculation of hamming distance.

Thus there are three dimension spaces of parameter. The simple process is mentioned below:

$$
\begin{aligned}
& \text { set all } X[x, y, z]=0 \\
& \text { for each }(a, b) \text { where } f(a, b)>s \\
& \text { for all } x \text { and } y \\
& z=\sqrt{\left((a-x)^{2}+(b-y)^{2}\right)} \\
& X[x, y, z)=X[x, y, z]+1
\end{aligned}
$$

The segmentation of image is used to reside boundaries and objects in images. The process of segmentation is a difficult and essential step in the system of image processing. Segmentation is a technique needed to exclude and separate the artifacts as well as residing the region of circular IRIS. The outer and inner boundaries of IRIS are estimated using segmentation (Samarati et al., 2009).

The segmented image is shown in Fig. 2.

\section{Canny Edge Detection}

The canny edge detector is one of the most commonly used tools of image processing predicting edges in a robust way (Zhu et al., 2000). The canny edge detection algorithm is known too much as an optimal edge detector. The algorithm has five steps namely smoothing, predicting gradients, non-maximum suppression, double thresholding and tracking edge by hysteresis.

The Fig. 3 shows the phase demodulator which is a 4 quadrant plane that represents the resulting matrix of feature from using the normalized image to Gabor filter, to binary code. This representation relies on the sign of both the imaginary and real part of feature matrix. The vertical axis indicates the imaginary tool with positive to up whereas the horizontal axis indicates the real tool with the positive to right (Biswas and Sil, 2012).

\section{Normalization}

After the segmentation of image and deciding the area of IRIS must be isolated from total picture. The process of normalization will generate regions of IRIS which have similar constant dimensions so that two pictures of similar IRIS under varied conditions will have feature characteristics at same spatial location. To recognize and contrast the area of IRIS the circular IRIS needed to be transformed to coordinate that have a fixed dimensions. This feature makes the comparison practically. The two main techniques used to retrieve features from iris image are Gabor filter and Daugman method. The Gabor filter is Gaussian's modulated by complex function of sinusoid (Palmer-Brown et al., 2009). The below figure shows the Gabor filter equation:

$$
\begin{aligned}
& \varphi(a, b, r f, \theta)=\frac{\omega 0}{\sqrt{2 \pi j}} e_{\delta j 2}^{\omega 02}(4(a \cos \cos \theta+b \sin \sin \theta) 2 \\
& +(-a \sin \sin \theta+b \cos \cos \theta) 2) \\
& e^{d(\omega 0 a \cos \cos \theta+\omega 0 b \sin \sin \theta)}-e^{-j 2 / 2}
\end{aligned}
$$

The Generator of Gabor filter is depicted in Fig. 4. The Daugman method is used for normalization stage. Daugman process performs in a way that offers points in the IRIS transformed to pair with corresponding points in polar coordinates in which $\theta$ is the angular field and $r$ is the radial distance (Vargahan et al., 2011). The normalized image is shown in Fig. 5.

\section{Denoising}

After normalization stage this study denoised the normalized image to develop the quality of it and also manage with possible noise that is added to image. In this study the normalized images are denoised using Contourlet transform. Contourlet is an isolated unidirectional 2D transform that is used to explain delicate details and curves in images. Contourlet transform effectively indicates those flat contours that are the major tools of every usual image (Zali-Vargahan et al., 2012). The Fig. 6 depicts the denoised image:

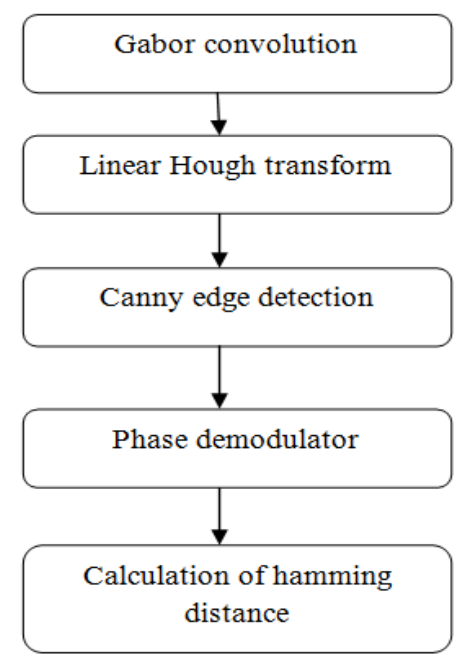

Fig. 1. Implementation of steps 


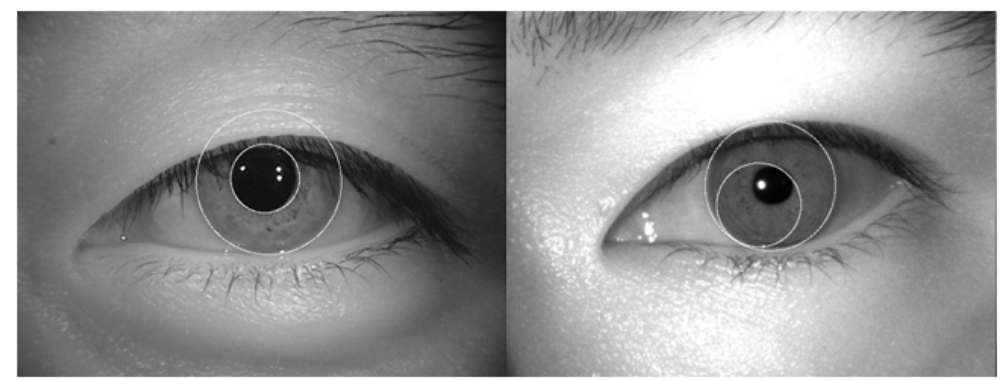

Fig. 2. Segmented image

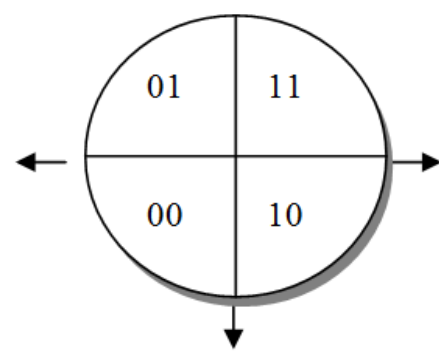

Fig. 3. Phase demodulator

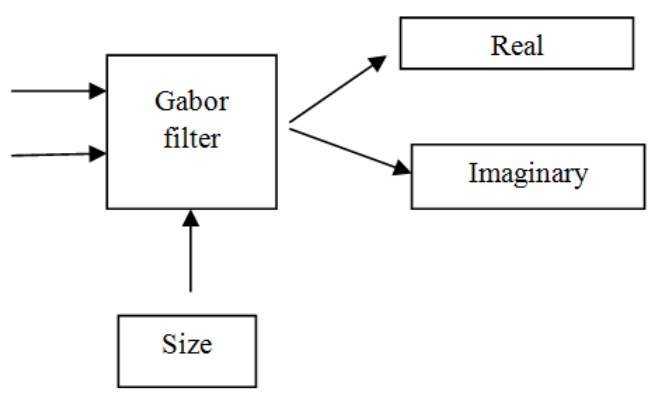

Fig. 4. Gabor filter generator

\section{Feature Extraction}

The essential IRIS features must be encoded as that contrast between templates can be made. Most systems of IRIS recognition make use of a band pass decomposition of the IRIS image to create a biometric template. Iris offers abundant information of texture a feature vector is formed which comprises of ordered feature sequences retrieved from different representation ofIRIS images. The Fig. 7 depicts the Laplacian of Gaussian image:

The Laplacian is a two dimensional isotropic estimation of image's second spatial derivative (Hussain and Agarwal, 2015). The image Laplacian highlights the rapid intensity change areas and is always used for edge detection. The Laplacian is always applied to an image that has been smoothed with approximating a Gaussian smoothing filter to decrease its sensitivity to noise.

\section{Iris Code Matching}

The last stage is to match the IRIS code. The templates of two IRIS code are compared by computing the hamming distance. Hamming distance is a fractional estimation of the number of bits disagreeing between two binary patterns. The hamming distance is a number used to represent the difference between two strings of binary numbers. It is a small part of a wider set of formulas used in analysis of information. Hamming's formulas permit PC to correct and detect mistakes on their own. For example the Hamming distance $d(a, b)$ between two vectors_a, b $\mathrm{C} \mathrm{f}$ (c) is the coefficient number in which they vary for instance:

- $\quad$ in $\mathrm{F}_{2}{ }^{(5)} \mathrm{d}(00111,11001)=4$

- $\quad$ in $\mathrm{F}_{3}{ }^{(4)} \mathrm{d}(0122,1220)=3$

- d fulfills the usual metric conditions:

- $d(a, b) \geq 0$ and $d(a, b)=0$ if and only if $a=b$

- $d(a, b)=d(b, a)$

- $\mathrm{d}(\mathrm{a}, \mathrm{c}) \leq \mathrm{d}(\mathrm{a}, \mathrm{b})+\mathrm{d}(\mathrm{b}, \mathrm{c})$ for any $\mathrm{a}, \mathrm{b}, \mathrm{c} \in \mathrm{F}(\mathrm{c})$

\section{Results and Discussion}

Iris recognition is a quickly developing biometric authentication method that uses techniques of pattern recognition on IRIS images to distinctly recognize an individual. The results of this study are depicted separately. The Fig. 8 shows the Gabor conversion and Gabor lines.

The Fig. 9 is the test image and recognized Fig. 8. The next resultant Fig. 10 is the after segmentation test and noise test.

The Table 1 summarizes various existing approaches for IRIS detection and their corresponding accuracy rates. There is no comparison of the common IRIS recognition metrics. As discussed in the earlier sections, in the present approach Gabor convolution has been used to filter the images. Linear Houghtransforms and canny edge detection has been used to segment the IRIS figures. Further the features have been subtracted from the figures and phase demodulator has been used encode the IRIS and save them as a template in a database file for IRIS recognition. 


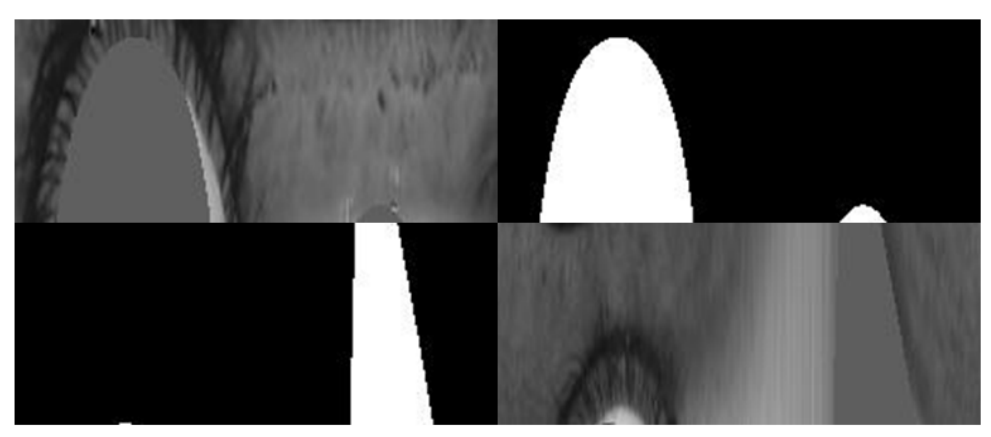

Fig. 5. Normalized image

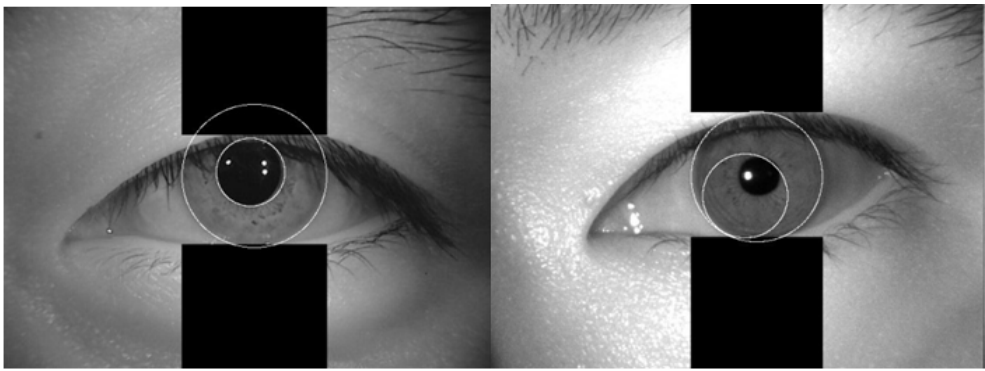

Fig. 6. Denoised image

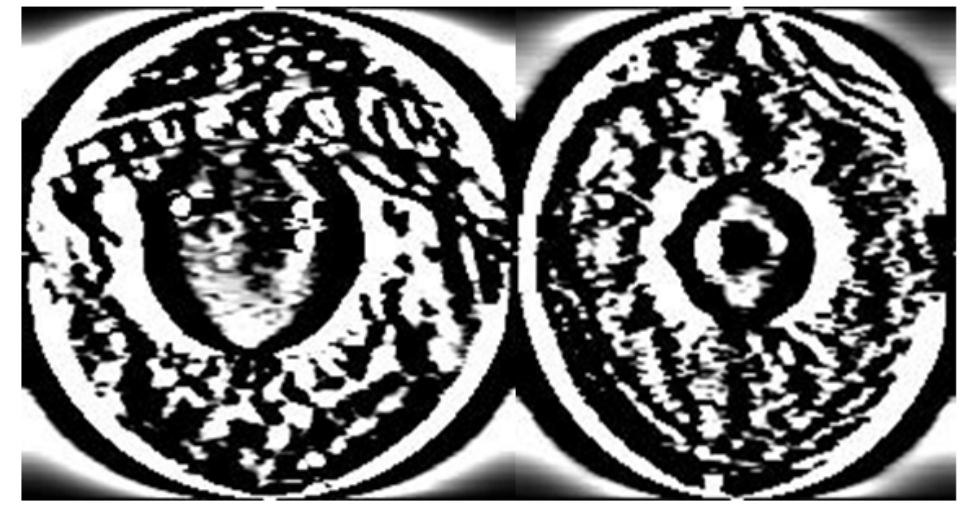

Fig. 7. Laplacian of Gaussian image
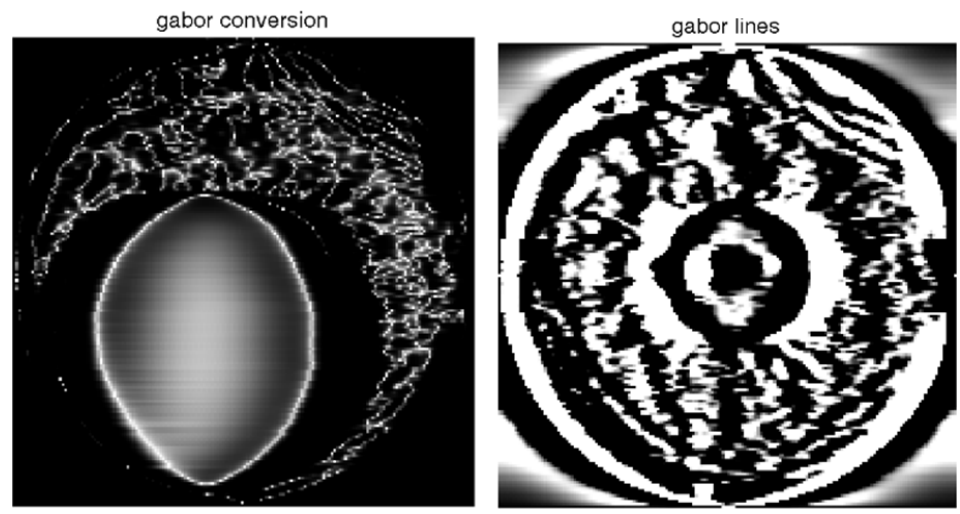

Fig. 8. Gabor conversion and Gabor lines 

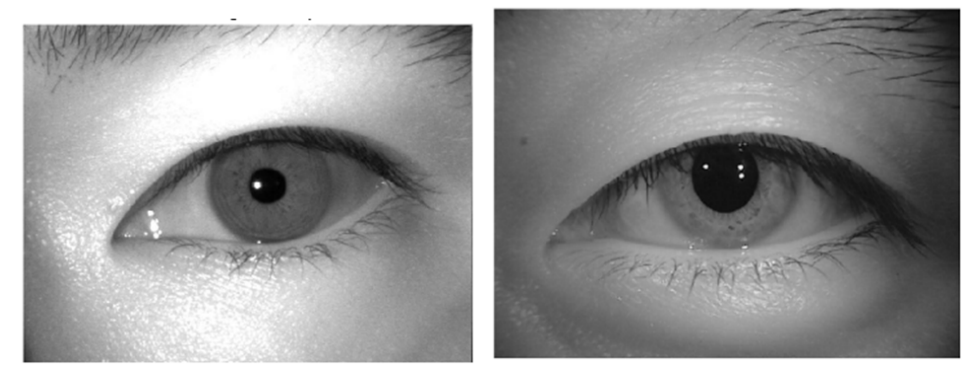

Fig. 9. Test and recognized image
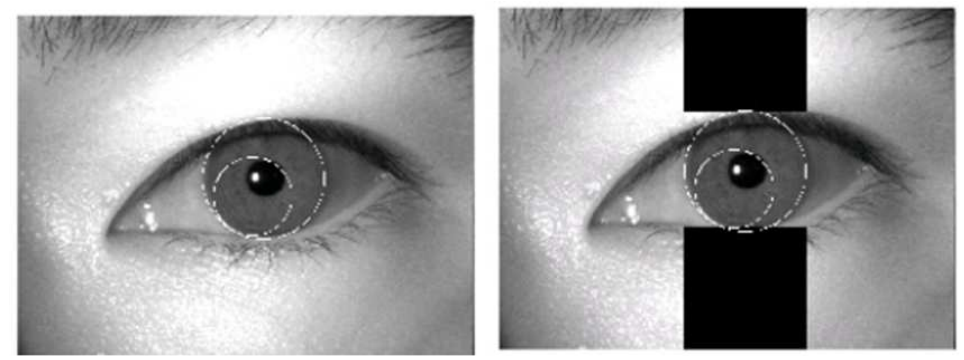

Fig. 10. After segmentation and noise test

Table 1. Technique or algorithm for iris detection

\begin{tabular}{|c|c|c|c|}
\hline Technique or algorithm for IRIS detection & Author & Year & Accuracy \\
\hline Phase based image matching & Miyazawa et al. & 2008 & $\begin{array}{l}\text { Calculated using state of art } \\
\text { DSP technology }\end{array}$ \\
\hline Hierarchical phase based matching & Durai and Karnan & 2010 & $\begin{array}{l}\text { Calculated using Fourier } \\
\text { Transform }\end{array}$ \\
\hline Daughman's Algorithm & Verma et al. & 2012 & $\begin{array}{l}\text { Calculated using Hamming } \\
\text { distance }\end{array}$ \\
\hline Canny edge detection and K-Means Algorithm & Jayachandra and Reddy & 2013 & calculated using K-means \\
\hline ICA, PCA, Daugman's Rubber Sheet Model & Chitte et al. & 2012 & $\begin{array}{l}\text { calculated using pattern } \\
\text { recognition }\end{array}$ \\
\hline Haar Wavelet Transform & Yao et al. & 2014 & $\begin{array}{l}\text { calculated using Euclidean } \\
\text { distance }\end{array}$ \\
\hline $\begin{array}{l}\text { The proposed approach \{Gabor convolutions, Linear } \\
\text { Hough transforms, Canny edge detection and } \\
\text { phase demodulator encoding\} }\end{array}$ & Mansour & 2016 & $\begin{array}{l}\text { Hamming distance } \\
\text { calculation }\end{array}$ \\
\hline
\end{tabular}

The Hamming distance of the encoded images is calculated to while doing the IRIS recognition to retrieve the IRIS related information in a faster pace thereby finding the most similar IRIS figure from the database accurately.

It can be very clearly seen that the approach adapted in this study yields results that are far better than that of the existing techniques.

\section{Conclusion}

In this study the author has proposed as well as implemented an effective and rapid real time Gauss Laplace algorithm for segmenting and localizing the pupil and IRIS boundaries of eye from database images. The algorithm predicts the boundaries and center reliably and accurately despite of the presence of eyelashes under reduced interface of contrast and in the occurrence of excess illumination. This study has proposed an IRIS recognition system that exhibits greater performance when compared with that of the previously available techniques. The author has also compared the accuracy of the proposed approach based on Gauss Laplace filters with different existing approaches. The outcomes have shown that the proposed approach has relatively quicker time of execution than that of the existing approaches.

\section{Recommendations for Future}

In future the researchers can propose some other new algorithms for recognition of IRIS. The future 
work would be to recognize IRIS from a larger database that has huge volume of information. Further new techniques can be proposed and tested for different variations of the input from size and illumination. The author also suggests that the future researchers can come up with algorithms for recognition of IRIS using minimal hardware and less expensive cameras such that IRIS recognition is done in a cost effective manner.

\section{Acknowledgment}

I thank two anonymous reviewers for constructive comments that helped me improve this manuscript.

\section{Ethics}

This article is original and contains unpublished material. The corresponding author confirms that all of the other authors have read and approved the manuscript and no ethical issues involved.

\section{References}

Alotaibi, S. and A. Hebaishy, 2014. Using phase demodulator for encoding iris features. Proceedings of the International Conference on Advances in Computing, Communications and Information Technology, (CIT' 14).

Biswas, R. and J. Sil, 2012. An improved canny edge detection algorithm based on type- 2 fuzzy sets. Proc. Technol., 4: 820-824. DOI: $10.1016 /$ j.protcy.2012.05.134

Chitte, P., G. Rana, R. Bhambare, A. More and A. Kadu et al., 2012. IRIS recognition system using ICA, PCA, Daugman's rubber sheet model together. Int. J. Comput. Technol. Electr. Eng., 2: 16-23.

Chouhan, B. and S. Shukla, 2010. Analysis of statistical feature extraction for Iris Recognition System using Laplacian of Gaussian filter. Int. J. Applied Eng. Res., 1: 528-535.

Durai, C.D. and M. Karnan, 2010. Iris recognition using modified Hierarchical Phase-Based Matching (HPM) technique. IJCSI, 7: 43-48.

Hussain, Z. andD. Agarwal, 2015.A comparative analysis of edge detection techniques used in flame image processing. Int. J. Adv. Res. Sci. Eng., 4: 3703-3711.

Jayachandra, C. and H.V. Reddy, 2013. Iris recognition based on pupil using canny edge detection and kmeans algorithm. Int. J. Eng. Comput. Sci., 2: 221-225.

Joshi, R., 2004. Application of the Hough transform to modeling the horizontal component of road geometry and computing heading and curvature.
Ma, L., Y. Wang and T. Tan, 2002. Iris recognition using circular symmetric filters. National Laboratory of Pattern Recognition, Institute of Automation, Chinese Academy of Sciences.

Mansour, R.F., 2015. Using adaptive mutation to accelerate the convergence of immune algorithms for prediction of 3D molecular structure. Int. J. Comput. Applic., 37: 127-133. DOI: $10.1080 / 1206212 X .2016 .1188568$

Miyazawa, K., K. Ito, T. Aoki, K. Kobayashi and H. Nakajima, 2008. An effective approach for IRIS recognition using phase-based image matching. IEEE Trans. Patt. Anal. Mach. Intell., 30: 1741-1756. DOI: 10.1109/TPAMI.2007.70833

Palmer-Brown, D., C. Draganova, E. Pimenidis and H. Mouratidis, 2009. Engineering Applications of Neural Networks.1st Edn., Springer Science and Business Media, New York, ISBN-10: 3642039693 , pp: 496.

Samarati, P., M. Yung, F. Martinelli and C. Ardagna, 2009. Information Security: 12th International Conference.1st Edn., Springer, New York, pp: 144.

Savithiri, G. and A. Murugan, 2010. Iris recognition technique using Gaussian pyramid compression. International Conference on Recent Trends in Business Administration and Information Processing, Mar. 26-27, Springer, Kerala, India, pp: 325-331. DOI: 10.1007/978-3-642-12214-9 52

Schmid, N., M. Ketkar, H. Singh and B. Cukic, 2006. Performance analysis of iris-based identification system at the matching score level. IEEE Trans. Inform. Forens. Security, 1: 154-168. DOI: $10.1109 /$ TIFS.2006.873603

Tisse, L., L. Martin, L. Torres and M. Robert, 2003. Person identification technique using human iris recognition. J. Syst. Res., 4: 67-75.

Vargahan, Z.B., M.C. Amirani and H. Seyedarabi, 2011. Fast iris recognition. J. World's Electr. Eng. Technol., 2: 04-08.

Verma, P., M. Dubey, P. Verma and S. Basu, 2012. Daughman's algorithm method for IRIS recognition-a biometric approach. Int. J. Emerg. Technol. Adv. Eng., 2: 177-185.

Yao, W., Z. Jun, Y. Feng and M. Jun, 2014. Iris feature extraction based on Haarwavelet trans form. Int. J. Security Applic., 8: 265-272. DOI: 10.14257/ijsia.2014.8.4.24

Zali-Vargahan, B., C. Amirani and H. Seyedarabi, 2012. Contourlet transform for iris image segmentation. Int. J. Comput. Applic., 60: 41-44.

Zhu, Y, T. Tan and Y. Wang, 2000. Biometric personal identification based on Iris Patterns.Proceedings of the 15th International Conference on Pattern Recognition, Sept. 3-7, IEEE Xplore Press, Barcelona, pp: 801-804. DOI: 10.1109/ICPR.2000.906197 